\title{
S3A: UM SISTEMA DE AVALIAÇÃO DA APRENDIZAGEM ATIVA
}

Juliana de Santana Silva - julisantana26@hotmail.com

UFBA, Programa de Pós-Graduação em Mecatrônica

Rua Aristides Novis 02, Federação, $2^{\circ}$ andar

40210-630 - Salvador - Bahia

Herman Augusto Lepikson - hlepikson@gmail.com

UFBA, Programa de Pós-Graduação em Mecatrônica

Rua Aristides Novis 02, Federação, $2^{\circ}$ andar

40210-630 - Salvador - Bahia

Armando Sá Ribeiro Junior - asrj@ufba.com

Universidade Federal da Bahia, Escola Politécnica

Rua Aristides Novis 02, Federação

40210-630 - Salvador - Bahia

Resumo: Nos últimos anos, o uso de métodos ativos de aprendizagem vem se destacando nos cursos de engenharia. Apesar dos benefícios promovidos por estes métodos, há desafios associados à avaliação da aprendizagem nos aspectos complexidade e precisão. Este trabalho propõe o Sistema de Avaliação da Aprendizagem Ativa - S3A como modelo de avaliação da aprendizagem apto a analisar o desenvolvimento das competências dos alunos em diversos contextos, com ênfase no processo. O S3A foi implementado integrando a metodologia Problem Based Learning - PBL com a metodologia convencional (aulas expositivas e avaliação de conteúdo), nos cursos de engenharia e arquitetura oferecidos por uma instituição pública, utilizando como métrica de análise os conceitos de: (i) eficiência: relação custo - benefício;(ii) eficácia: análise do alcance de metas; (iii) efetividade; análise das transformações (contribuições potenciais). Este modelo avaliou o desenvolvimento de 12 competências nos contextos de aprendizagens colaborativa, autônoma, direcionada, por erro $e$ de autoavaliação. Este estudo concluiu que o S3A apresenta como benefícios $(i)$ um modelo de avaliação que viabiliza análise do desenvolvimento de competência em contextos diversos, (ii) permite o acompanhamento, análise de perfil do aluno, (iii) é acoplável aos instrumentos disponíveis de avaliação e métodos ativos, (iv) apresenta parâmetros que viabilizam a análise da aprendizagem individual em contextos de projetos em grupo e precisão no conhecimento adquirido de modo especificado. Por outro lado, o custo devido ao tempo de implementação foi considerado alto. Além disso, houve limitações experimentais e temporais, o que sugere trabalhos futuros para automatização e testes com amostras maiores.

Palavras-chave: Avaliação dinâmica. Aprendizagem ativa. Aprendizagem autônoma. 


\section{INTRODUÇÃO}

O manejo de diversas metodologias ativas é uma das principais tendências dos modelos educacionais atuais (PUNCREOBUTR, 2016). No Brasil, especificamente nos cursos de engenharia, novas diretrizes curriculares propõem, entre as diversas modificações, a inserção do ensino ativo (BRASIL, 2019).

A capacidade de promover aprendizagem em contexto de colaboração, do modo autônomo e através dos erros são os principais benefícios dos métodos ativos (ROCHALEMOS, 2014). Apesar do potencial de benefícios que estas abordagens podem promover, a avaliação destas aprendizagens ainda é um desafio, destacando (i) complexidade de avaliação da aprendizagem individual com desenvolvimento de projetos em grupo; (ii) imprecisão no conhecimento de memória sobretudo de teorias avançadas (RIBEIRO, 2010).

Nesse contexto, este trabalho objetivou propor, implementar e analisar um modelo de avaliação da aprendizagem para métodos ativos tendo sua natureza dinâmica e integrada como principais características.

\section{REFERENCIAL TEÓRICO}

Não há uma definição formal na literatura para as Metodologias Ativas (MAs), no entanto, são apresentados diversos elementos para caracterizar e enquadrar as diversas metodologias como MAs (YAMAMOTO, 2016). A fundamentação principal das MAs é a caracterização do aluno como o centro do processo e o principal responsável pela busca e construção do seu conhecimento, enquanto que o professor atua como facilitador e estimulador do processo de aprendizagem (ROCHA; LEMOS, 2014). A aprendizagem pela interação social de Lev Vygotsky (1896-1934), aprendizagem pela experiência John Dewey (1859-1952), aprendizagem significativa (consideração dos conhecimentos prévios) de David Ausubel (1918-2008) e aprendizagem autônoma de Paulo Freire (1921-1997) são também consideradas fundamentações da MA (DIESEL et.al.,2017)

A Aprendizagem Baseada em Problemas (WOOD, 2003), Aprendizagem Baseada em Projetos (KOLMOS; DE GRAAFF, 2014.), Aprendizagem Baseada em Equipe (LAMM et al 2014), Aprendizagem Baseada em Redes Sociais (WU, 2014), Aprendizagem Baseada em Dispositivos Móveis (HERLINAH; HABIBI, 2016), Aprendizagem por Pares (AŞIKSOY; ÖZDAMLI, 2016) e Ensino Sob Medida (OLIVEIRA et. al., 2015) são exemplos do arsenal de métodos propostos na literatura.

Apesar dos benefícios promovidos, os métodos ativos apresentam desafios associados à avaliação da aprendizagem. Uma avaliação da aprendizagem é definida como uma análise dos dados provenientes do processo de ensino e aprendizagem e são categorizadas em: (i) estática: confere ênfase ao resultado, verificando o que e quanto o aluno aprendeu (ii) dinâmica: foca no processo de aprendizagem, avaliando a capacidade de aplicar os conhecimentos (RABELO; 2001; SACRISTÁN, 2009).

Além dos tipos de avaliações, há diversos instrumentos de avaliação. Macdonald e SavinBaden (2004) apresentam alguns instrumentos que são utilizados com sucesso na Aprendizagem Baseada em Problemas (Problem Based Learning - PBL), entre eles: apresentação em grupo, avaliação tripartida, salto triplo, autoavaliação, avaliação dos pares, exames orais, jogos reflexivos, relatórios e textos retalhos. A despeito das abordagens de avaliação, Ribeiro (2010) destaca como desafio a complexidade da avaliação individual e a precisão do conhecimento adquirido. 


\section{S3A - SISTEMA DE AVALIAÇÃO DA APRENDIZAGEM ATIVA}

O S3A objetiva avaliar o desenvolvimento de competências nos diversos contextos de aprendizagem possibilitados pelas metodologias ativas. Este modelo de avaliação da aprendizagem é definido como: (i) dinâmico por considerar o processo além dos resultados finais e; (ii) integrado: por utilizar as avaliações para reorientar processos de aprendizagem, conforme a Figura 1.

Figura 1 - S3A: Sistema de Avaliação da Aprendizagem Ativa

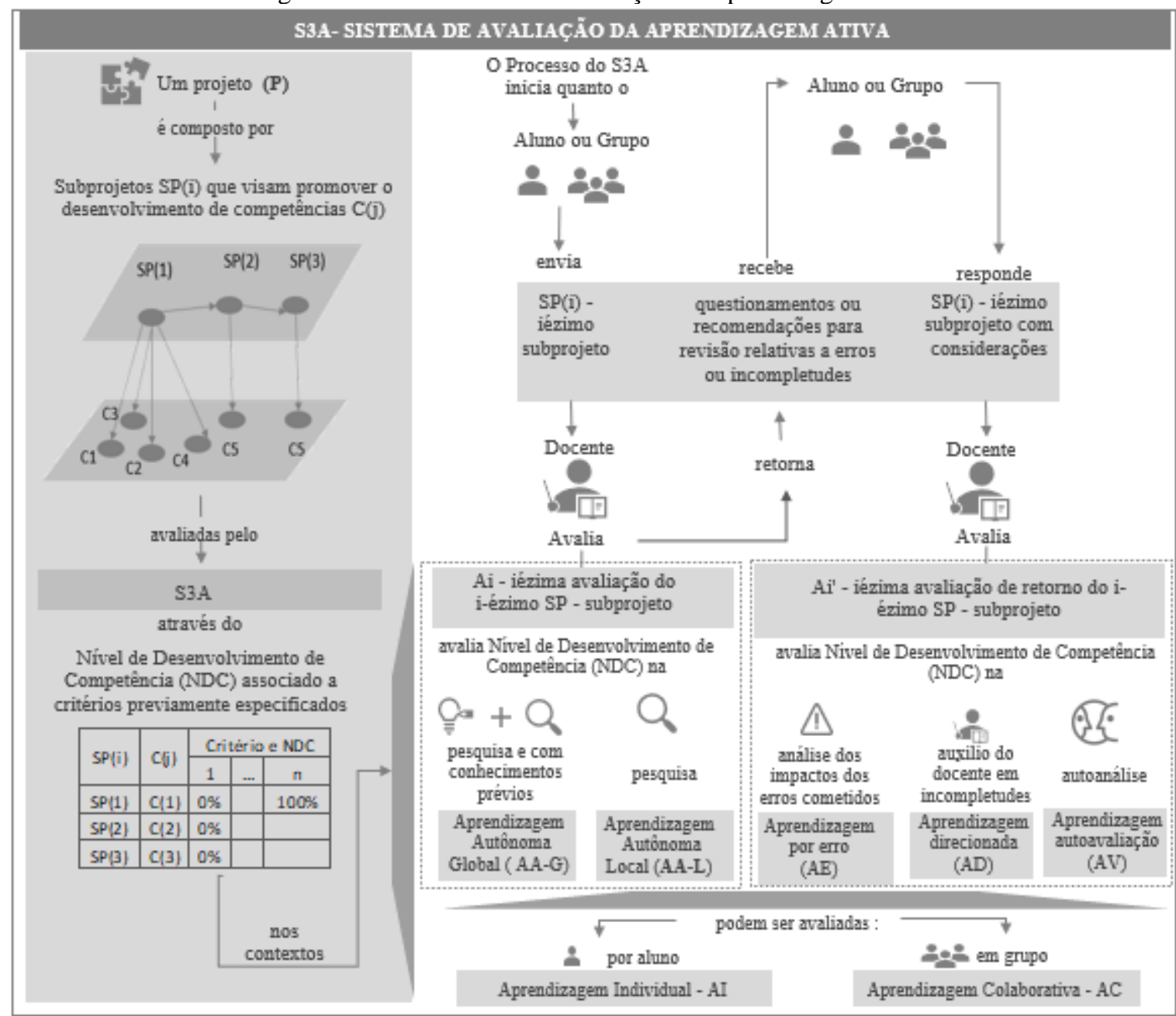

Fonte: Autoria Própria

O projeto $(P)$ é um planejamento de aprendizagem materializado em uma atividade, projeto ou problema conforme a metodologia adotada. $\mathrm{P}$ é definido por subprojetos $(S P)$ que, de modo integrado, visam desenvolver um conjunto de competências. O processo de avaliação se inicia quando o aluno entrega um i-ézimo subprojeto $-S P_{i}$. O docente recebe o $S P_{i}$ e retorna questionamentos e recomendações relativas a erros ou incompletudes no projeto. $\mathrm{O}$ aluno retorna o $S P_{i}$ com considerações, de forma recursiva.

Nesse processo, uma avalição visa medir um Nível de Desenvolvimento de Competência - NDC que é um percentual, variante de 0 a 100\%, associado ao desenvolvimento de determinada competência no contexto de aprendizagem, conforme critérios previamente especificados. Os contextos avaliados estão relacionados, no S3A, como a aprendizagem 


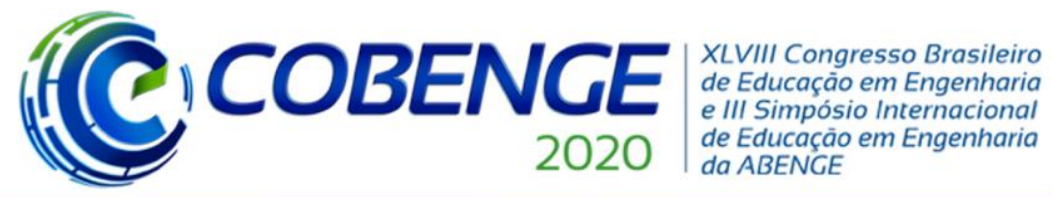

"Os desafios para formar hoje o engenheiro do amanhã"

autônoma local, autônoma global, por erro, direcionada ou autoavaliação descritos na Quadro 1.

Quadro 1 - Contextos de aprendizagem considerados pelo S3A

\begin{tabular}{|l|l|l|}
\hline COD & CONTEXTO & DESCRIÇÃO \\
\hline AA-L & $\begin{array}{l}\text { Aprendizagem } \\
\text { Autônoma Local }\end{array}$ & $\begin{array}{l}\text { avalia o desenvolvimento da atividade com mobilização de } \\
\text { conhecimentos adquiridos com a pesquisa. }\end{array}$ \\
\hline AA-G & $\begin{array}{l}\text { Aprendizagem } \\
\text { Autônoma Global }\end{array}$ & $\begin{array}{l}\text { avalia o desenvolvimento da atividade como um todo envolvendo a } \\
\text { mobilização dos conhecimentos prévios e que foram pesquisados. }\end{array}$ \\
\hline AE & $\begin{array}{l}\text { Aprendizagem por } \\
\text { Erro }\end{array}$ & $\begin{array}{l}\text { avalia a análise critica dos desdobramentos e impactos diversos de } \\
\text { um determinado erro cometido no projeto }\end{array}$ \\
\hline Aprendizagem & $\begin{array}{l}\text { avalia o desenvolvimento da atividade com os direcionamento em } \\
\text { apão de incompletudes relativas as dificuldades dos alunos. }\end{array}$ \\
\hline Autondizagem por & $\begin{array}{l}\text { avalia a autoanálise do desenvolvimento de competência pelo } \\
\text { próprio aluno. }\end{array}$ \\
\hline AI & $\begin{array}{l}\text { Aprendizagem } \\
\text { Individual }\end{array}$ & análise da aprendizagem em contexto individual \\
\hline AC & $\begin{array}{l}\text { Aprendizagem } \\
\text { Colaborativa }\end{array}$ & análise da aprendizagem em contexto coletivo \\
\hline
\end{tabular}

Fonte: Autoria Própria

Conforme o processo da Figura 1, a primeira avaliação $A_{i}$ analisa dois tipos de aprendizagens: a aprendizagem autônoma global $\left(A A_{g}\right)$ e local $\left(A A_{L}\right)$. Enquanto a segunda avaliação $A_{i}^{\prime}$ analisa as aprendizagens direcionadas, por erro e autoavaliação. Estas avaliações, definidas pelas Equações (1) e (2), podem ser avaliadas em grupo, individual ou em ambas.

$$
\begin{gathered}
A_{i}=\sum_{j=1}^{n} N D C_{j}\left(A A_{L}\right)+\sum_{j=1}^{n} N D C_{j}\left(A A_{g}\right) \\
A_{i}^{\prime}=\sum_{j=1}^{n} N D C_{j}(A E)+\sum_{j=1}^{n} N D C_{j}(A D)+\sum_{j=1}^{n} N D C_{j}(A V)
\end{gathered}
$$

Os diversos contextos de aprendizagens podem ser associados a coeficientes que definem o seu grau de importância relativo. Estes coeficientes podem revelar aspectos importantes: a relação entre coeficiente de $A_{i}$ e $A_{i}^{\prime}$ é uma taxa de recuperação associado a reavaliações dos alunos. A diferença entre AI que antecedem e sucedem a AC pode revelar o nível de uniformidade da aprendizagem em grupo.

\subsection{Aplicação do método}

O S3A foi implementado usando a metodologia PBL em disciplinas da área de resistência dos materiais, integrada à metodologia instrutivista (aulas expositivas e avaliação de conteúdo), conforme expressa a Figura 2. A aprendizagem individual foi avaliada pelo modelo tradicional, enquanto o S3A avaliou a aprendizagem colaborativa e nos outros contextos especificados na tabela 1. As métricas para análise utilizadas foram os conceitos de eficiência, eficácia e efetividade, descritos a seguir. 
Figura 2 - Contexto de Implementação do S3A

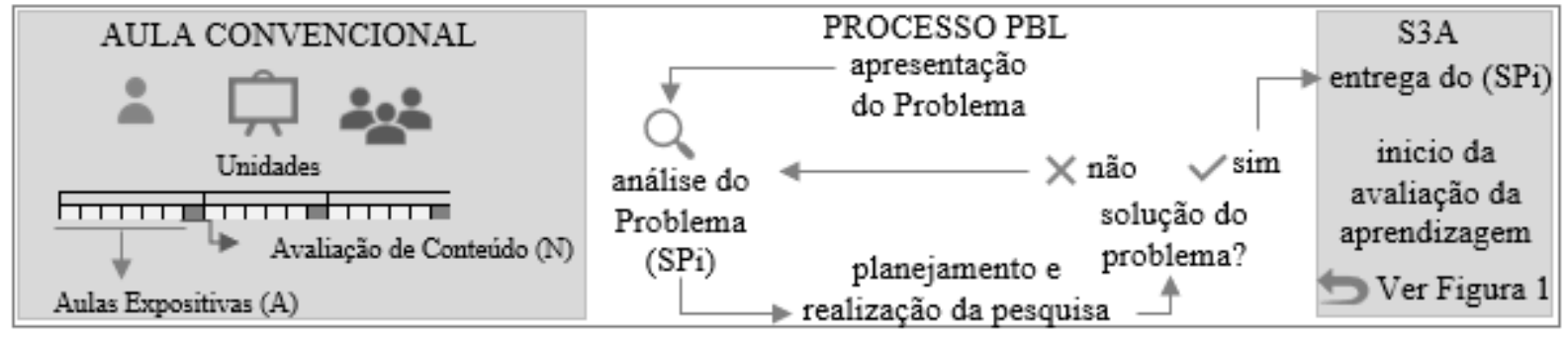

Fonte: Autoria Própria

\subsection{Ambiente, amostra e métricas de análise da implementação}

O S3A foi aplicado em uma universidade pública, a Universidade Federal da Bahia. Nesta instituição os cursos são estruturados em aulas expositivas com avaliação por unidades. O S3A e o PBL foram aplicados com adesão voluntária dos alunos em ambos cursos, focando em projeto de estruturas para um reservatório de água para combate a incêndio para cursos de engenharia e projeto de galpão industrial para os cursos de arquitetura.

Como métrica de análise, foram utilizados os conceitos de: (i) eficiência: dimensão expressa a relação do processo com o meio, avaliando a relação do custo e benefício do processo analisado (ii) eficácia: é uma medida que analisa o resultado, o quão se alcançou as metas; (iii) efetividade: é uma dimensão relacionada capacidade de transformação promovida pelo processo.

\section{RESULTADOS E DISCUSSÕES}

O projeto teve adesão de $91 \%$ nos cursos de arquitetura e de $50 \%$ nos cursos de engenharia. Foram avaliadas 5 Competências Técnicas - CT e 7 Competências Não Técnicas CNT, com políticas de avaliações distintas, conforme a Figura 3. No curso de engenharia a política de adesão envolveu o acréscimo da avaliação do projeto as demais avaliações, enquanto, no curso de arquitetura a política envolveu a possibilidade de realizar a substituição de uma avaliação convencional realizada.

Figura 3 - Amostra, política de adesão e competências consideradas na implementação do S3A

\begin{tabular}{|c|c|c|c|}
\hline \multicolumn{2}{|c|}{ AMOSTRA } & \multicolumn{2}{|r|}{ COMPETÊNCIAS } \\
\hline \multirow{3}{*}{ 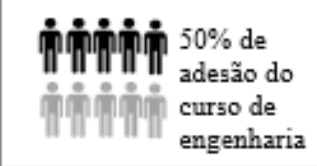 } & \multirow{3}{*}{$\begin{array}{l}91 \% \text { de } \\
\text { adesão do } \\
\text { curso de } \\
\text { arquitetura }\end{array}$} & $\mathrm{CT1}$ & Capacidade de analis ar cus to \\
\hline & & $\mathrm{CT} 2$ & Capacidade de analis ar aspectos de segurança \\
\hline & & CT3 & Capacidade de determinar diagrama de corpo livre \\
\hline \multirow{2}{*}{\multicolumn{2}{|c|}{ POLÍTICA DE ADESÃo }} & CT4 & Capacidade de determinar es forç os intemos solicitantes \\
\hline \multirow{2}{*}{\multicolumn{2}{|c|}{ Engenharia: $\Sigma$ Acréscimo (N5 -Projeto) }} & CT5 & Capacidade de avaliar o es tado tensões \\
\hline & & CNT1 & Capacidade de realizar pesquis a \\
\hline \multirow{3}{*}{\multicolumn{2}{|c|}{$\begin{array}{l}N_{F}=0,15 \cdot N_{1}+0,25 \cdot N_{2}+0,2 \cdot N_{3}+0,4 \cdot N_{4} \\
N_{F}=0,125 \cdot N_{1}+0,208 \cdot N_{2}+0,167 \cdot N_{3}+0,333 \cdot N_{4}+0,167 \cdot N_{4}\end{array}$}} & CNT2 & Capacidade de apren dizagemautônoma (AA-bcal) \\
\hline & & $\mathrm{CNT3}$ & Capacidade de planejamento \\
\hline & & CNT4 & Capacidade de análise critica de projetos \\
\hline \multicolumn{2}{|c|}{ Arquitetura: $\leftrightarrows$ Substituição (Ni) } & CNT5 & Capacidade de análise dos requisitos de projeto \\
\hline \multirow{2}{*}{\multicolumn{2}{|c|}{$N_{F}=0,3 \cdot N_{1}+0,3 \cdot N_{2}+0,4 \cdot N_{3}$}} & CNT6 & Competências relacionadas a comunicação oral \\
\hline & & $\mathrm{CNT} 7$ & Competências relacionadas a comunicação es crita \\
\hline
\end{tabular}

Fonte: Autoria Própria 


\subsection{Aprendizagem por problemas}

A aplicação do S3A na avaliação da aprendizagem por problemas permitiu avaliar o NDC em contextos diversos, conforme a Figura 4.

Figura 4 - NDC (AP) - Nível de Desenvolvimento de Competência na Aprendizagem por Problemas

Equipe 01 - ARQ

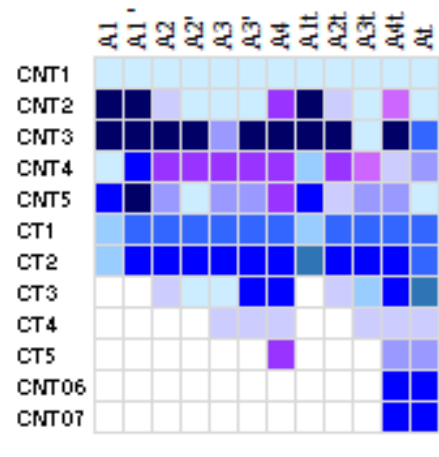

$\operatorname{NDC}(A P)=67 \% \quad a=0,7 \quad b=0,3$

Equipe 04 - ARQ

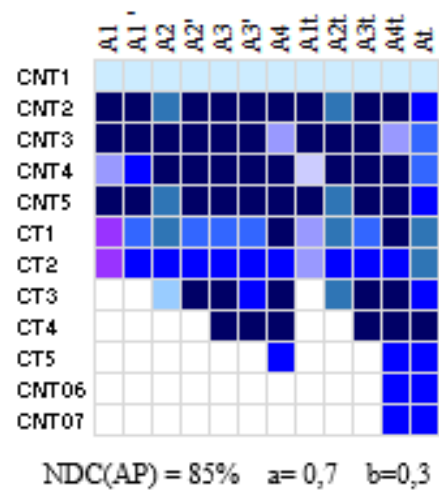

Equipe 02 - ARQ

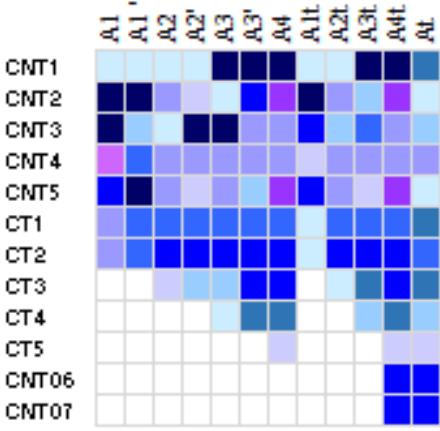

$\mathrm{NDC}(\mathrm{AP})=69 \% \quad \mathrm{a}=0,7 \quad \mathrm{~b}=0,3$

Equipe 01 - ENG

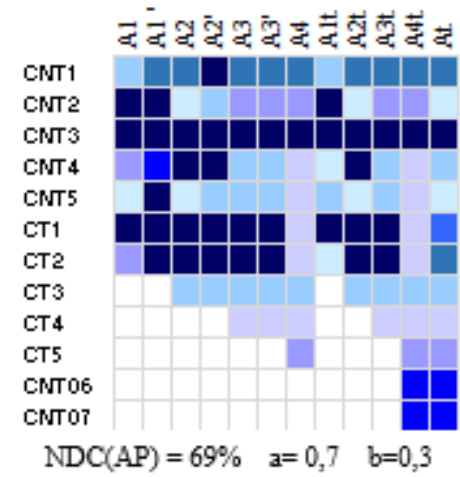

Equipe 03 - ARQ

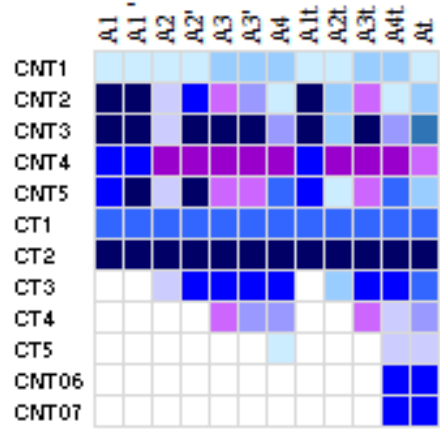

$\mathrm{NDC}(\mathrm{AP})=67 \% \quad a=0,7 \quad b=0,3$

Equipe 02 - ENG

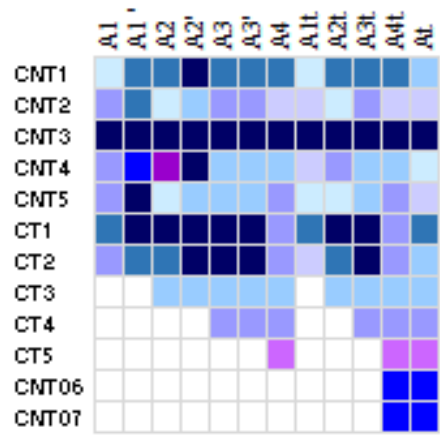

$\mathrm{NDC}(\mathrm{AP})=63 \% \quad \mathrm{a}=0,7$

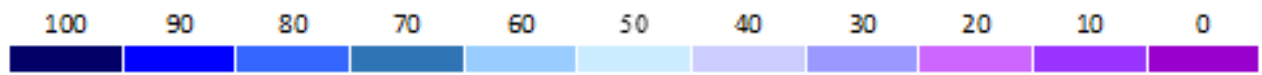

Nota: ENG - engenharia; ARQ - Arquitetura; Ai - iézima avaliação; Ai'-iézima avaliação de retorno; CNT Competência Não Técnica (vide Figura 3); CT- Competência Técnica (vide Figura 3); Ait = aAi + bAi’; a coeficiente de aprendizagem autônoma; b -coeficiente de aprendizagem por erro, direcionada e autoavaliação.

Fonte: Autoria Própria

Este modelo de avaliação viabilizou a identificação: (i) de quais competências foram menos desenvolvidas e em quais contextos. Exemplo: nota-se a possibilidade de análise do desenvolvimento de CT03 - Capacidade de analisar esforços internos de forma autônoma ou por direcionamentos, erros e autoavaliação ao longo do processo; (ii) da evolução de uma determinada competência ao longo dos subprojetos entregues. Exemplo: as equipes de arquitetura apresentam NDC baixo no início, mas foram melhorando, enquanto, as equipes de engenharia apresentaram NDC médios e estáveis; (iii) perfil de nível de desenvolvimento de competências de uma determinada turma, aluno ou equipes. Exemplo: o NDC de engenharia e arquitetura foi em média $60 \%$, apenas uma equipe superou essa faixa, apresentado um NDC (AP) de $85 \%$. Apesar de a maioria das equipes apresentar desempenhos próximos, notou-se muitas diferenças nas competências que mais foram desenvolvidas nos cursos. 


\subsection{Aprendizagem autônoma}

A aprendizagem autônoma local informa o quanto o aluno consegue aplicar conteúdos através de pesquisa. Já a autônoma global informa o quanto o aluno consegue aplicar conteúdos pesquisados e já vistos em sala. A comparação entre permite analisar a influência das aulas no processo de aplicação, conforme ilustra Figura 5. A equipe 04 de arquitetura, em A1, A3 e A4, apresentou AA - local de 100\%, isso significa que conseguiu aplicar $100 \%$ dos conteúdos não apresentados em sala de aula de modo satisfatório. Mas as outras competências que envolveram a necessidade de mobilizar conhecimentos apresentados em sala e pesquisados não foram muito bem desenvolvidas, resultando em um AA - global inferior ao local das avaliações.

Figura 5 - NDC (AA) - Nível de Desenvolvimento de Competência na Aprendizagem Autônoma

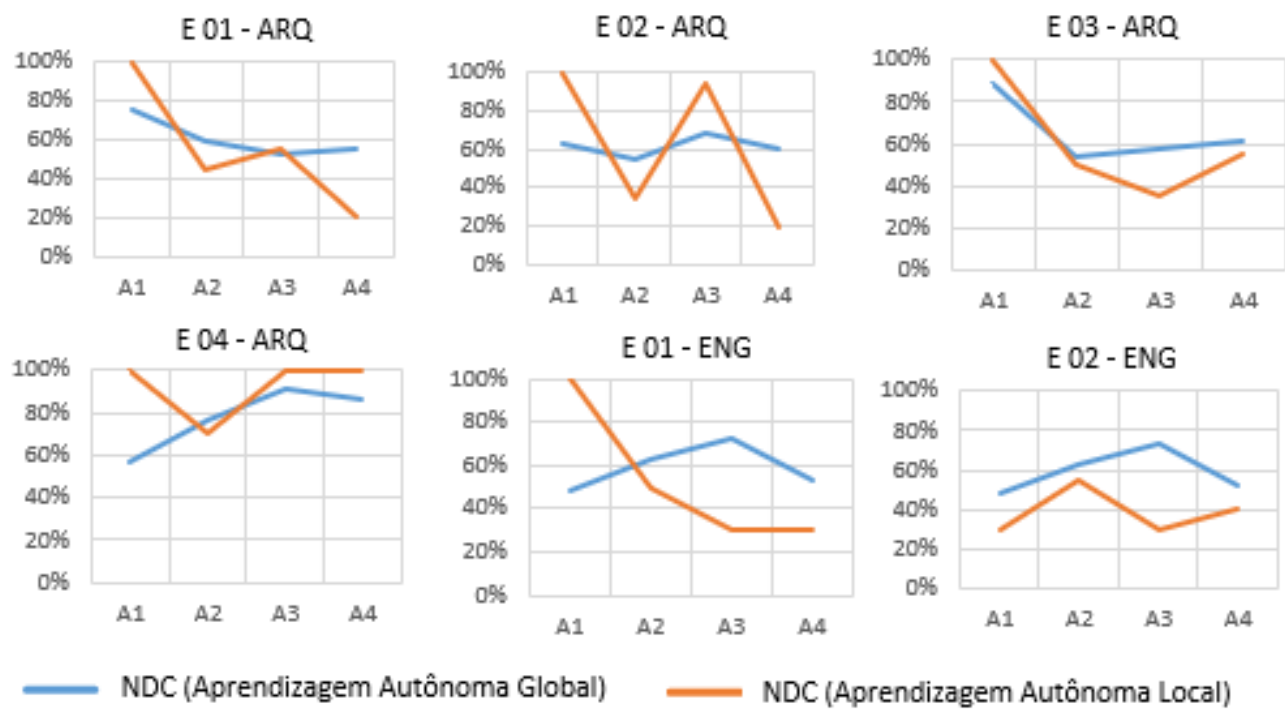

Nota: E- equipes; ARQ- arquitetura; ENG-engenharia; Ai - i ézima avaliação

Fonte: Autoria Própria

\subsection{Aprendizagem direcionada, por erro e autoavaliação}

A avaliação $A_{i}^{\prime}$ ilustrada na Figura 4, foi avaliada a aprendizagem (i) Direcionada: em razão de incompletudes sinalizadas e relacionadas a dificuldades, foi elaborado o guia interativo com acessos a compreensão de como se realizava cada subprojetos, com exemplos similares; (ii) erro: avaliou os retornos das correções e a análise dos impactos econômicos e de segurança de um subdimensionamento e superdimensionamento, por exemplo; (iii) autovaliação: autopercepção das competências não desenvolvidas e dificuldades sinalizadas ao longo do projeto.

\subsection{Aprendizagem individual e colaborativa}

A aprendizagem individual foi avaliada por meios tradicionais, com avaliação de conteúdo, enquanto a colaborativa foi avaliada através do S3A, com os respectivos desempenhos apresentados na Figura 6. Como foram avaliadas em contextos distintos o nível de uniformidade de aprendizagem colaborativa não foi mensurado. As avaliações convencionais ocorrem por unidades não especificando quais as competências desenvolvidas. 
Figura 6 - Desempenho dos alunos nas aprendizagens convencional e por problemas

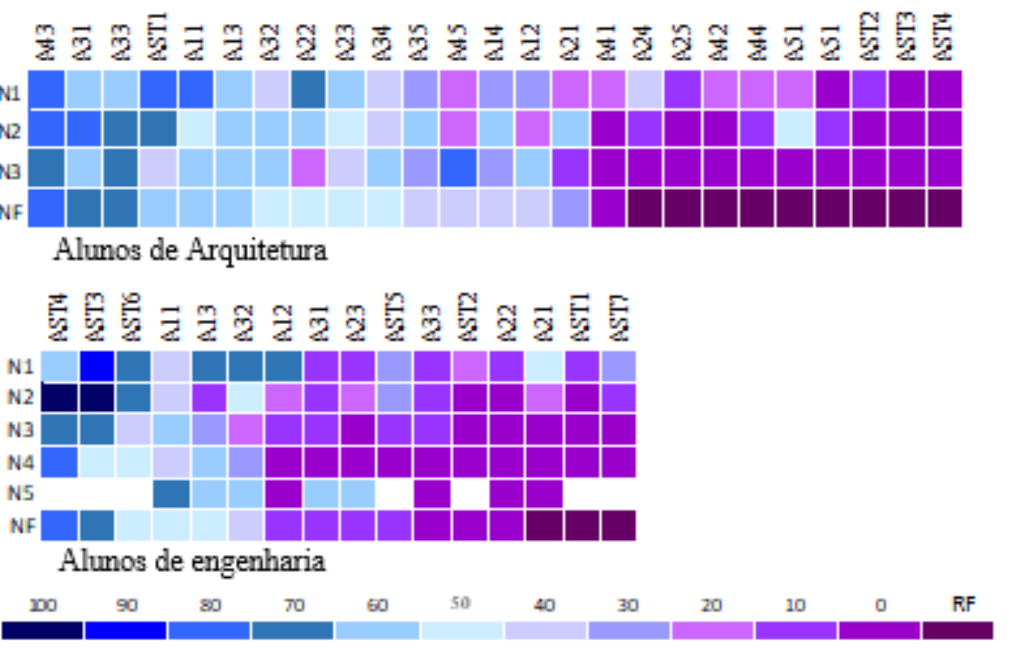

Nota: Ni - i-ézima avaliação; NF- avaliação final (vide Figura 3); Aij - j-ézimo aluno da i-ézima equipe; ASTj - j-ézido aluno que não aderiu ao projeto; RF - Reprovação por Falta.

Fonte: Autoria Própria

\subsection{Análise da eficácia, efetividade e eficiência absoluta e relativa}

O Quadro 2 apresenta uma análise da: (i) eficiência: análise dos benefícios e custos; (ii) eficácia: análise do alcance das metas (objetivos do S3A) e; efetividade: análise das transformações - $\mathrm{T}$ (contribuições as lacunas da literatura).

Quadro 2 - Análise da eficiência, eficácia e efetividade S3A

\begin{tabular}{|c|c|c|}
\hline MÉTRICA & & DESCRIÇÃO \\
\hline \multirow{7}{*}{$\begin{array}{l}\text { Eficiência: } \\
\text { Relação entre o } \\
\text { Benefício-B e os } \\
\quad \text { custos-C }\end{array}$} & B-1 & $\begin{array}{l}\text { Viabiliza a análise dos desenvolvimentos de competências nos contextos diversos de } \\
\text { aprendizagem. }\end{array}$ \\
\hline & B-2 & Permite acompanhar a evolução de uma determinada competência ao longo de todo o curso. \\
\hline & B-3 & $\begin{array}{l}\text { Permite analisar o perfil de desenvolvimento de competência de alunos, grupos e turmas, } \\
\text { identificando quais foram as competências menos desenvolvidas e em quais contextos. }\end{array}$ \\
\hline & B-4 & É acoplável a outras metodologias ativas, tais como as citadas no tópico Referencial Teórico. \\
\hline & B-5 & $\begin{array}{l}\text { É possível integrar a outras abordagens de avaliação, tais com as citadas no tópico } \\
\text { Referencial Teórico. }\end{array}$ \\
\hline & B-6 & $\begin{array}{l}\text { Através do coeficiente de uniformidade de aprendizagem colaborativa permite a análise da } \\
\text { uniformidade de aprendizagem dos alunos nas equipes. }\end{array}$ \\
\hline & $\mathrm{C}-1$ & Custo horário alto quando comparado as demais abordagens de avaliação. \\
\hline \multirow{2}{*}{$\begin{array}{l}\text { Eficácia: análise } \\
\text { do alcance de } \\
\text { metas-M }\end{array}$} & M1 & Dinâmico: considera a evolução no processo e não apenas o resultado \\
\hline & M2 & Integrado: reorienta as práticas educacionais. \\
\hline \multirow{2}{*}{$\begin{array}{l}\text { Efetividade: } \\
\text { análise das } \\
\text { transformações- } \\
\text { T }\end{array}$} & $\mathrm{T} 1$ & $\begin{array}{l}\text { A diferença entre os NDCs de aprendizagens individuais de uma determinada competência } \\
\text { que antecedem e sucedem o NDC de aprendizagem colaborativa desta mesma competência } \\
\text { tem potencial em avaliar o nível de uniformidade na aprendizagem colaborativa (em grupo), } \\
\text { contribuindo com o desafio da avaliação da aprendizagem individual no grupo, citado por } \\
\text { Ribeiro (2010). }\end{array}$ \\
\hline & $\mathrm{T} 2$ & $\begin{array}{l}\text { A especificação da competências e conteúdos, habilidades e atitudes com verificação deles } \\
\text { nos contextos diversos pode contribuir com o desafio citado por Ribeiro (2010) como } \\
\text { imprecisão em conhecimentos em teorias avançadas. }\end{array}$ \\
\hline
\end{tabular}




\section{RESULTADOS E DISCUSSÕES}

O S3A é um modelo de avaliação desenvolvido como consequência da aplicação de uma abordagem de gestão para aprendizagem ativa desenvolvida pelos autores deste trabalho.

Apesar dos benefícios promovidos pelo S3A os custos de operacionalização foram considerados altos em função do esforço demandado. Por outro lado, estes custos podem ser reduzidos com aplicações sucessivas em uma determinada disciplina, em razão do reaproveitamento da estruturação das avaliações em competências. Além disso, o estudo foi dotado de algumas limitações experimentais associadas ao período (teste em apenas um semestre) e amostral (número pequeno de amostras).

A despeito destas limitações, o S3A se demostrou efetivo na sua implementação, demostrando sua contribuição para a avaliação (i) da uniformidade da aprendizagem individual em contextos de desenvolvimento de projetos em grupo bem como (ii) do desenvolvimento de competências em contextos diversos tais como, autônoma, direcionada, autoavaliação e sobretudo por erro, normalmente não considerados pelas avaliações convencionais.

\section{Agradecimentos}

Á FAPESB - Fundação de Amparo A Pesquisa do Estado da Bahia pelo apoio na forma de bolsa de pesquisa.

\section{REFERÊNCIAS}

AŞIKSOY, G.; ÖZDAMLI, F. Flipped Classroom adapted to the ARCS Model of Motivation and applied to a Physics Course. Eurasia Journal of Mathematics, Science \& Technology Education, v. 12, n. 6, 2016

BRASIL. Ministério da Educação. Resolução CNE/CES n² de 24 de abril de 2019. Institui as Diretrizes Curriculares Nacionais do Curso de Graduação em Engenharia. Disponível em: http://portal.mec.gov.br/conselho-nacional-de-educacao/atos-normativos--sumulas-parecerese-resolucoes/33371-cne-conselho-nacional-de-educacao/74471-resolucoes-cne-ces-2019. Acessado em: 2 ago 2019

DIESEL, A.; BALDEZ, A. L. S.; MARTINS, S. N. Os princípios das metodologias ativas de ensino: uma abordagem teórica. Revista Thema, v. 14, n. 1, p. 268-288, 2017.

HERLINAH, H.; HABIBI, B. Technological Innovation of Learning Mobile-Based Learning. In: Proceeding International Conference on Mathematics, Science, Technology, Education and their Applications. 2016.

KOLMOS, A.; DE GRAAFF, E. Problem-based and project-based learning in engineering education. Cambridge handbook of engineering education research, p. 141-161, 2014.

LAMM, M. H.; DORNEICH, M.; ROVER, D.T. Team-Based Learning in Engineering Classrooms: Feedback Form and Content Adds Value to the Learning Experience. 2014.

MACDONALD, Ranald; SAVIN-BADEN, Maggi. A briefing on assessment in problembased learning. LTSN, 2004. 
OLIVEIRA, V.; VEIT, E. A.; ARAUJO, I. S. Relato de experiência com os métodos Ensino sob Medida (Just-in-Time Teaching) e Instrução pelos Colegas (Peer Instruction) para o Ensino de Tópicos de Eletromagnetismo no nível médio. Caderno brasileiro de ensino de física. Florianópolis. Vol. 32, n. 1 (abr. 2015), p. 180-206, 2015.

PUNCREOBUTR, V. Education 4.0: new challenge of learning. St. Theresa Journal of Humanities and Social Sciences, v. 2, n. 2, 2016.

RIBEIRO, L. R. C. Aprendizagem baseada em problemas: uma experiência no ensino superior. São Carlos: Edufscar, 2008.

ROCHA, H. M.; LEMOS, W. M. Metodologias ativas: do que estamos falando? Base conceitual e relato de pesquisa em andamento. IX Simpósio Pedagógico e Pesquisas em Comunicação. Resende, Brasil: Associação Educacional Dom Boston, v. 12, 2014.

WOOD, D. F. ABC of learning and teaching in medicine: Problem based learning. BMJ: British Medical Journal, v. 326, n. 7384, p. 328, 2003.

WU, T.T. Using smart mobile devices in social-network-based health education practice: A learning behavior analysis. Nurse education today, v. 34, n. 6, p. 958-963, 2014.

\title{
S3A: AN ACTIVE LEARNING ASSESSMENT SYSTEM
}

\begin{abstract}
In recent years, the use of active learning methods has been highlighted in engineering courses. Despite the benefits promoted by these methods, there are challenges associated with assessing learning in terms of complexity and precision. This work proposes the Active Learning Assessment System - S3A as a model for learning assessment capable of analyzing the development of students' competence in different contexts, with emphasis on the process. The S3A was implemented by integrating the Problem Based Learning - PBL methodology with the conventional methodology (expository classes and content evaluation), in the engineering and architecture courses offered by a public institution, using the concepts of: (i) efficiency: cost - benefit (ii) efficacy: analysis of the goals achieved; (iii) effectiveness; analysis of transformations (potential contributions). This model evaluated the development of 12 competences in the contexts of collaborative, autonomous, directed, error and selfassessment learning. This study concluded that S3A presents as benefits (i) an evaluation model that allows analyzing the development of competences in different contexts, (ii) allows monitoring, an analysis of the student's profile, (iii) it is integrable with the instruments of available evaluation and active methods, (iv) presents parameters that allow analyzing individual learning in contexts of group projects and obtaining precision in the knowledge acquired in a specified way. However, the cost due to the time of implementation was considered high. In addition, there were some experimental and temporal limitations, which suggests new studies for automation and tests with larger samples.
\end{abstract}

Keywords: Dynamic assessment. Active learning. Autonomous learning. 